\title{
Modelling blockage effects using a spectral element method
}

\author{
M. D. Griffith* K. Hourigan* M. C. Thompson*
}

(Received 27 October 2004, revised 1 March 2005)

\begin{abstract}
We employ a two-dimensional spectral element method to investigate the effect of various semi-circular blockages on the laminar flow between two flat plates. This simplified geometry has a general relevance to blocked flows, but more academically, also relates to work on the backward-facing step, presenting a qualitatively similar flow with a single, independent step, or blockage, parameter. The parameter space for Reynolds number between 50 and 3000, and blockage ratio ranging from 0.05 to 0.9 is mapped out. The results with blockage ratio of 0.5 compare favourably with previous work on the flow over a backward-facing step, with the analysis suggesting that the onset of three-dimensionality in the flow is likely to be closely matched. Flow phenomena associated with blockage ratios above and below the customary half-blockage are summarised, including smaller than expected
\end{abstract}

*Dept. Mechanical Engineering, Monash University, Melbourne, Australia. martin.griffith@eng . monash. edu . au

See http://anziamj.austms.org.au/V46/CTAC2004/Grif for this article, (c) Austral. Mathematical Soc. 2005. Published April 21, 2005. ISSN 1446-8735 
flow separation lengths for lower blockages, and vortex shedding and unusual structures within the recirculation zones for higher blockages.

\section{Contents}

1 Introduction

2 Method

C170

2.1 Problem definition . . . . . . . . . . . . . C C170

2.2 Numerical method . . . . . . . . . . . . . . . . C171

3 Results

C173

3.1 Flow separation length . . . . . . . . . . . . . C173

3.2 Wake behaviour . . . . . . . . . . . . . . C174

4 Conclusions

C179

References

C179

\section{Introduction}

The study of blockage effects in internal flows has typically focused on determining correction factors for small blockage ratios in wind tunnels. The standard study of the flow over a backward-facing step closely matches a half-blocked flow, but such studies tend to be more interested in the various instabilities that occur in the flow, rather than on any effects the step height may have. This study examines the flow behaviour between two flat plates, with the addition of a semi-circular blockage, of variable size, attached to one side. The blockage ratio is defined as $b=r / D$, where $r$ is the radius of the semi-circular blockage and $D$ is the distance between the two plates. Figure 1 


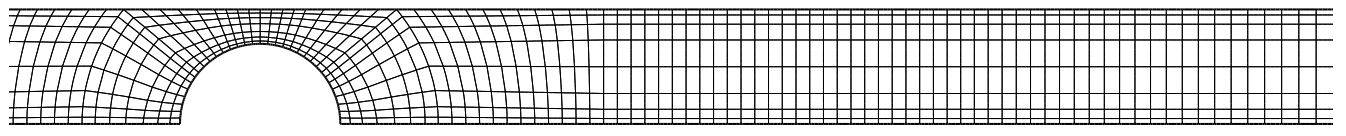

Figure 1: Computational mesh, showing macro-elements, used for blockage ratio 0.7 .

shows the macro-elements for a portion of one of the computational meshes used, with a blockage ratio of 0.7 . Such a study has particular relevance to the flow through arterial constrictions, but also to industrial applications involving pipe flow.

The subject of this study bears much similarity to the flow over a backward-facing step, of which there is a far greater availability of literature. The standard test-section geometry is well-known, consisting of a channel with a sudden expansion. This expansion causes an immediate separation of the flow, along with the appearance of a recirculation zone. The geometry of the present study differs in the existence of a constriction of the flow before the expansion, resulting in a skewed velocity profile over the bump, and also a separation point dependent on Reynolds number, rather than on the geometry, as is the case with the backward-facing step. The work of Armaly et al. [1] offers a detailed review of the subject, including an experimental investigation with a fixed expansion ratio of 0.516 , an aspect ratio of 18:1 and Reynolds numbers ranging through the laminar, transitional and turbulent regimes. With increasing Reynolds number in the laminar regime, the length of the first recirculation zone also increased, and was accompanied by the appearance, downstream, of more recirculation zones on alternating sides of the channel. Three-dimensional effects were also observed.

Numerical studies of the problem $[2,3,4]$ give good accounts of the development of unsteadiness and three-dimensionality in the flow. The work of Williams and Baker [8] is particularly useful in its comparison of two and three-dimensional numerical simulations of backward-facing step flow. For the geometry used in the present two-dimensional study, the exact onset of 
three-dimensionality to the flow is unknown and must be kept in consideration. An investigation focusing on higher blockage effects was carried out by Sahin and Owens [5], but it used the geometry of a freely suspended cylinder between two plates. Although the geometry bears only a limited similarity to this study (it is symmetrical and creates a wake consisting of twin vortices) Sahin and Owens' work showed that the proximity of the walls would increase the critical Reynolds number at which shedding would occur.

This paper will firstly give a description of the method used in the study, followed by an outline of the various flow behaviours occurring over the parameter space.

\section{Method}

\subsection{Problem definition}

The aim of this work is to map out and describe the parameter space for the two-dimensional flow between flat plates, with the addition of a semi-circular blockage of varying size. Figure 1 shows a section of the macro-element mesh with $b=0.7$. Similar meshes were produced for blockage ratios ranging from 0.05 to 0.9 . The Reynolds number is based on the hydraulic diameter $2 D$ :

$$
\mathrm{Re}=\frac{2 D U_{\mathrm{ave}}}{\nu},
$$

where $U_{\text {ave }}$ is the average flow velocity between the plates at inlet and $\nu$ denotes the kinematic viscosity. Data cited from other studies are rescaled to fit this Reynolds number definition. For each simulation, the fluid is modelled as being uniform, homogeneous, incompressible and Newtonian. A time-constant, fully-developed Poiseuille profile, with a non-dimensionalised average velocity of 1 , and a peak of 1.5 , is prescribed at the inlet, such that $U=6 y(1-y)$, where the bottom and top walls are placed at $y=0$ and 
$y=1$, respectively. For the outlet boundary, the pressure is fixed and the normal velocity gradient is set to zero. A short region of high viscosity is applied just before the outlet to dissipate any strong vortices created when the fluid starts to flow from rest.

The two parameters governing the flow behaviour are the Reynolds number and the blockage ratio. Tests were done over a broad range of Reynolds number, with the main restriction being the higher velocities generated over the semi-circular blockage. Blockage ratios ranging from 0.05 to 0.9 were used. Runs were made for Reynolds numbers ranging from 40 to 3000; however, for higher blockages the upper limit was more restricted, with the maximum achievable Reynolds number at $b=0.9$ being $R e=500$. This highlights a limitation of the two-dimensional analysis. For the flow between two flat plates, the transition to turbulence is known to begin when $\operatorname{Re} \approx 2000$, with the constriction of the flow over the blockage also acting as an additional tripping mechanism. The unknown point of transition has implications for the assumptions of laminar flow and the Poiseuille velocity profile used at the inlet. This must be kept in consideration when interpreting the results, but also presents an avenue of further study.

\section{$2.2 \quad$ Numerical method}

The flow field was obtained from a numerical solution of the two-dimensional, time-dependent, Navier-Stokes equations, given here in primitive variable form, with the incompressibility constraint

$$
\begin{aligned}
& \frac{\partial \overline{\boldsymbol{u}}}{\partial t}+\overline{\boldsymbol{u}} \cdot \boldsymbol{\nabla} \overline{\boldsymbol{u}}=-\nabla p+\nu \nabla^{2} \overline{\boldsymbol{u}} \\
& \boldsymbol{\nabla} \cdot \overline{\boldsymbol{u}}=0
\end{aligned}
$$

where $\overline{\boldsymbol{u}}=(\bar{u}(t, x, y), \bar{v}(t, x, y))$ is the velocity vector and $p$ and $\nu$ are the kinematic pressure and viscosity. The spectral element method used to dis- 
cretize and solve the equations, has been previously used and validated in the prediction of wake flows past rings [6] and circular cylinders [7].

The spectral element code solves for the velocity and pressure fields over each time interval, using three steps. The steps deal with the convection, pressure and diffusion terms in equation (2). The nonlinear convection term is integrated forward in time using a third-order Adams-Bashforth scheme. The second step deals with the pressure term. Applying the continuity constraints to the divergence of the momentum equation yields a Poisson equation, which is then solved using a higher order pressure boundary condition. The third step involves the diffusion term. This is solved implicitly using a Crank-Nicolson scheme that is second-order accurate in both space and time.

A typical spectral element mesh, depicting the macro-elements, with blackage ratio $b=0.7$, is shown in Figure 1. For each element, the solution variables in each direction are approximated using high-order Lagrangian polynomial interpolants. Gauss-Legendre-Lobatto quadrature is used to evaluate the product of the integrals of the flow equations with the local weighting functions. The advantage of this approach is that only a limited number of element nodes contribute to the equations formed at a particular node, resulting in more efficient run times. The final grid resolution for most simulations was $49(7 \times 7)$ nodes per element. For higher blockages and Reynolds numbers, elements comprising of $8 \times 8$ nodes per element had to be employed, in order to properly resolve parts of the flow field. Increasing the nodal concentrations beyond the levels described produced no discernible effect on recirculation lengths or downstream vorticity profiles.

The inlet is placed six plate widths (6D) upstream of the blockage, and the outlet 25 widths downstream. Increasing the distance of the blockage beyond six plate widths from the inlet produced no effect. However, increasing the outlet length did have an effect on simulations run at higher Reynolds number and blockage ratio, with what resembled a convective instability forming near the outlet. This apparent instability would disappear if the outlet length was increased sufficiently, along with the commensurate increase in required 
Figure 2: Streamlines for $b=0.5$ and $\operatorname{Re}=1000$

computational time. However, further investigation revealed, importantly, that the occurrence of this instability, and its removal via increased outlet length, produced no effect on the immediate wake vicinity of the blockage.

\section{$3 \quad$ Results}

\subsection{Flow separation length}

Figure 2 shows the streamlines for the flow over a blockage of 0.5 , at $\operatorname{Re}=$ 1000 . This geometry is the closest to the classic backward-facing step case. For this case, two flow separation zones (FSZs) can be seen, with one on each wall. The initial FSZ is formed by the sudden expansion at the blockage, and the subsequent adverse pressure gradient along the back half of the blockage. The length $L$ of this initial flow separation zone serves as an effective way of characterising the flow behaviour. The end of the first recirculation zone was determined by locating where the line of zero velocity, emanating from the flow separation point at the blockage, met the lower wall. Dividing by the blockage height $r$ normalises the recirculation length for different blockage ratios.

For this blockage size and up to $\mathrm{Re} \approx 400$, the recirculation length matched well with the data of previous studies. Above this point, the two-dimensional computational results give a recirculation length lower than previous experimental results for a backward-facing step [1]. Williams and Baker [8] have examined this problem previously in their investigation of 
two and three-dimensional numerical simulations for backward-facing steps. They found that the flow became three-dimensional at $\operatorname{Re} \approx 400$, and that recirculation lengths calculated from these three-dimensional results, more closely matched the experimental results of Armaly et al. [1]. The onset of this three-dimensionality can be put down to the effect of the sidewalls of the experimental apparatus. Hence, regardless of any effect caused by the different two-dimensional geometries, a divergence between the experimental and two-dimensional results at this Reynolds number is not wholly unexpected.

Figure 3 shows a plot of all normalised recirculation lengths for all blockages. Also depicted on the graph are the results of Armaly et al. [1], up to $\operatorname{Re}=1200$, where they observed the transitional flow regime beginning. The divergence at $\operatorname{Re} \approx 400$ between the two data sets, at which the flow is thought to become three-dimensional, can be clearly seen, and bears a strong similarity to the comparisons made by Williams and Baker [8]. Excepting the lower blockage ratios, the data sets for each ratio appear to follow a similar curve, meaning the normalised recirculation length is largely a function of upstream Reynolds number.

The lower blockage ratios, $0.05,0.1$ and 0.2 , produced recirculation lengths lower than those for higher blockages. These results can be put down to the low fluid velocities at these blockage heights. If we look at the inlet velocity profile, at $y=0.05$, it returns $U_{y=0.05}=0.285 U_{\text {ave }}$. At these heights, the more rapid parts of the fluid flow over the blockage are largely unperturbed, while the low fluid velocity near the blockage results in a smaller recirculation zone. A similar effect is either masked or not reproduced at $b=0.9$, since the entirety of the fluid flow is forced through the tiny gap.

\subsection{Wake behaviour}

As observed in many backward-facing step studies, the appearance of a second FSZ on the upper wall, near the end of the initial FSZ, would occur as 


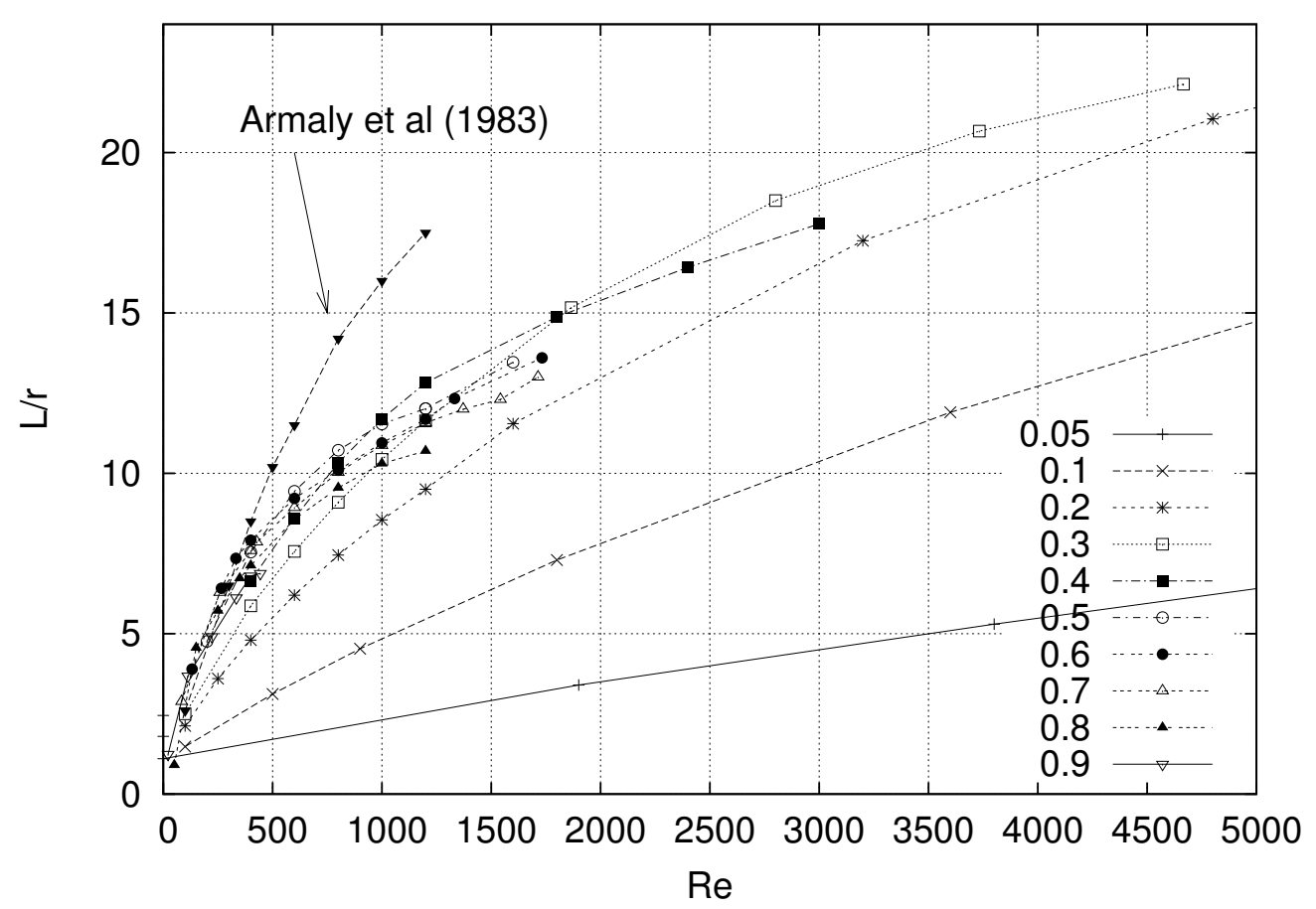

Figure 3: Plot of normalised recirculation length $L / r$ against upstream Reynolds number Re. 


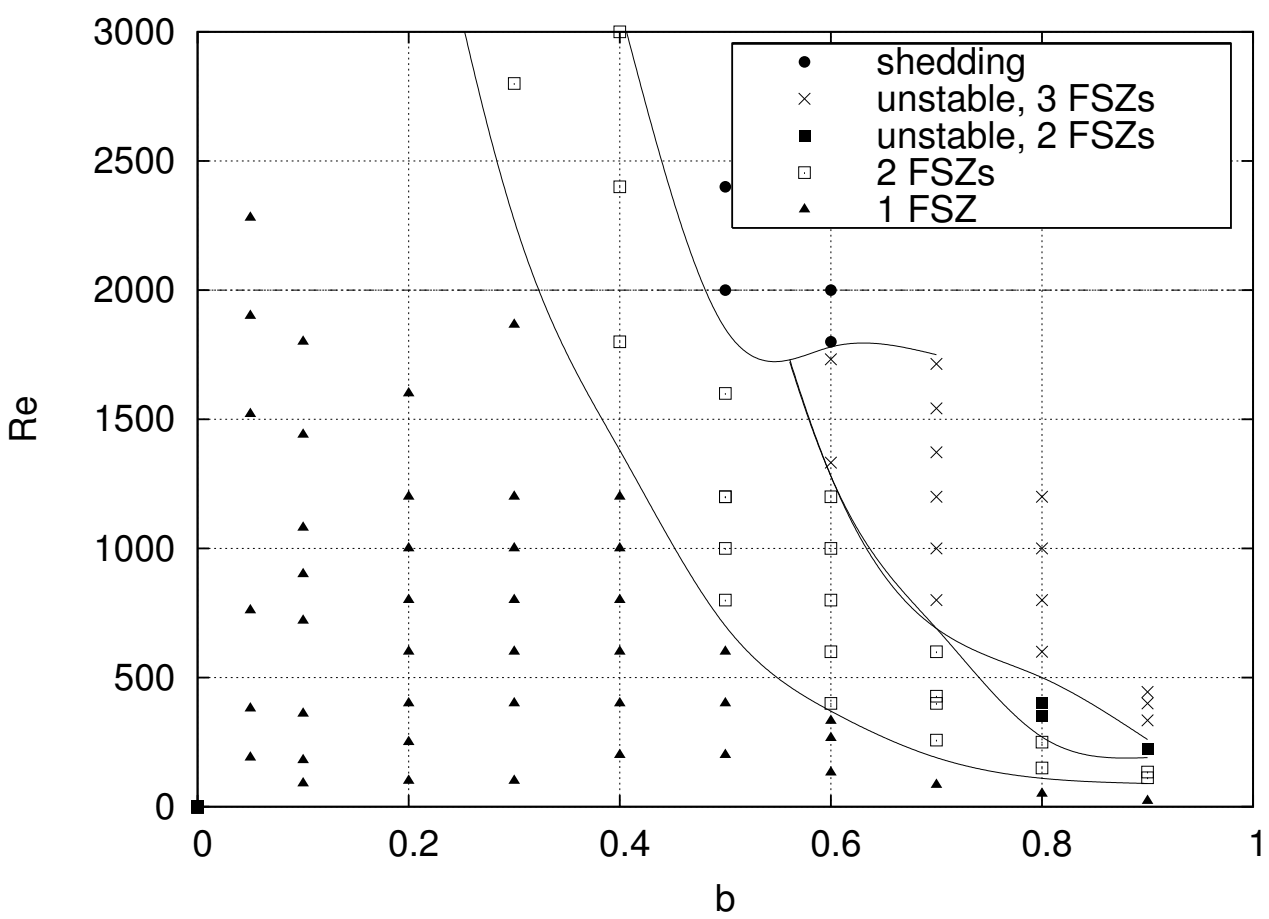

Figure 4: Map of wake behaviour over Reynolds number and blockage. Lines indicate the boundaries of each wake behaviour. Data points are also plotted.

the Reynolds number was increased. It was found that the secondary zone of recirculation could only be produced with blockages of 0.3 and greater. By a similar mechanism, a third zone of recirculation could be produced, but only for $b \geq 0.6$. Figure 4 presents a map of the parameter space. Those points labeled "unstable" denote the appearance at the end of the initial recirculation zone of a vortex structure, not observed in the previously cited backward-facing step investigations. This steady vortex structure is never apparent with the 0.5 blockage ratio, which is the closest geometry to the standard backward-facing step. The vortex structure makes a brief appear- 


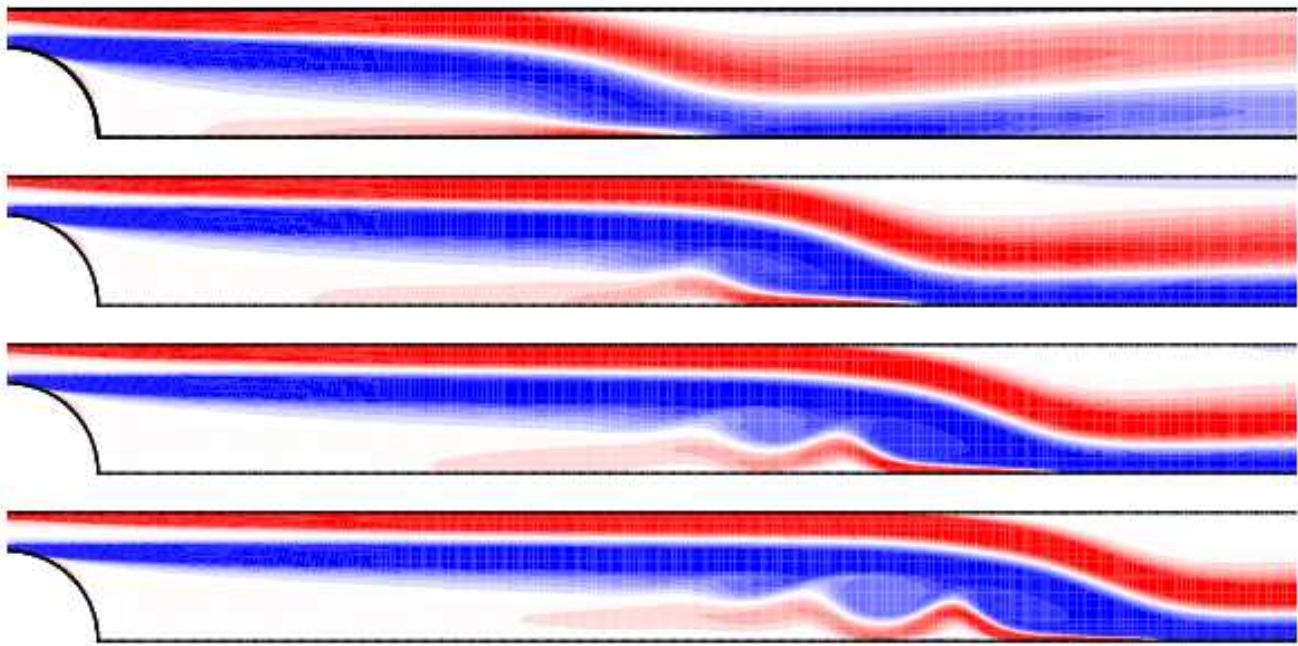

FiguRE 5: Visualisation of the vorticity field, at $b=0.7$ and $\operatorname{Re}=400,800$, 1200,1600 .

ance with the 0.6 blockage ratio, at $\operatorname{Re} \approx 1500$. The phenomenon is best observed with the 0.7 and 0.8 blockage ratios. Figure 5 shows plots of the vorticity at $b=0.7$ and $\operatorname{Re}=400,800,1200,1600$. The first instance, at $\operatorname{Re}=400$, is located below the relevant boundary on Figure 4, and the steady vortex structure is not immediately apparent. The next three instances clearly depict the appearance of the vortex structure at the end of the first recirculation zone. The structure seems to consist, initially, of a single smaller zone of recirculating flow, just behind the point at which the flow reattaches to the bottom wall. As the Reynolds number increases, a second smaller recirculating zone can be seen, particularly for $\mathrm{Re}=1200$. At $\operatorname{Re}=1600$, a third zone is apparent, indicating that a series of these zones is developing upstream, as the Reynolds number is increased.

The vortex structure here seems quite complex. At $R e=1600$, a series of three mini-recirculations is apparent, and upon a closer examination (not 
presented here) even the hint of a fourth is present. Closer inspection reveals the presence of further, even smaller recirculations, between the obvious ones in Figure 5, closer to the bottom wall. Figures 5 suggests that an increase in Reynolds number promotes the continued development of the vortex pattern, right up until the onset of vortex shedding from the blockage. Tests were performed and the steady state did not respond to small perturbations in the flow, higher mesh resolutions or longer outlet lengths.

The points labeled "shedding" on Figure 4 represent those simulations where the flow sheds vortices from the blockage. The different vortex dynamics, on and above the corresponding boundary, have not yet been fully analysed. In comparison to Sahin and Owens work [5], the onset of shedding is delayed to a much higher Reynolds number $(\mathrm{Re} \approx 1800$ rather than $\operatorname{Re} \approx 340$ ). This is to be expected, as there is only one large vortex forming behind the asymmetric blockage of this study, as compared to the far more unstable twin vortices behind the cylinder in Sahin and Owens' study. It is possible that the proximity of the wall opposite the bump has an effect on the onset of shedding, especially if we consider that the flow seems to shed more easily at $b=0.5$, than it does at $b=0.8$.

Given that Armaly et al. observed the transition to turbulence commencing at $R e=1200$ for a 0.5 blockage ratio on a backward-facing step [1], we could reasonably surmise that the critical Reynolds number for transition would be even lower for higher blockage ratios. A blockage's effectiveness as a tripping mechanism would only increase with the size of that blockage. Therefore, it is likely the instability in the initial flow separation zone, as shown in Figure 5, only occurs above where the transition to turbulence would occur. The instability may be an indication that the flow at these Reynolds number is no longer two-dimensional and perhaps the same vortex structure would not be reproduced in a three-dimensional simulation. Work on the similarities and differences between two and three-dimensional simulations for high blockage ratios, similar to that by Williams and Baker [8] on a backward-facing step, is not available and, for now, can only be estimated. 


\section{Conclusions}

A numerical investigation of the two-dimensional laminar flow over semicircular blockages has been carried out. The results for $b=0.5$ compared favourably with the experimental work of Armaly et al. [1] and the numerical simulations of Williams and Baker [8]. The lengths of the initial flow separation were closely matched with the experimental work, up to $\operatorname{Re} \approx 400$, where the onset of three-dimensionality is thought to occur. Excepting the smaller blockage ratios, for a constant upstream Reynolds number, the normalised recirculation lengths seemed to all behave similarly. For blockage ratios equal to or less than 0.2 , it was found that the low velocities around the blockages, close to the channel wall, resulted in a smaller initial recirculation zone.

For blockage ratios of 0.4 and greater, vortex shedding from the blockage was observed for higher Reynolds numbers. For blockage ratios of 0.6 and greater the appearance of an apparent instability at the end of the first recirculation was observed, before the onset of shedding. Whether the same vortex structure will be reproduced in any three-dimensional simulations or experiments is currently under investigation.

Acknowledgment: This research was principally supported by the Australian Research Council, by ARC Grant No. DP0452664

\section{References}

[1] Armaly, B. F., Durst, F., Pereira, J. C. F. and Schoenung B., Experimental and theoretical investigation of backward-facing step flow, J. Fluid Mech., 127, 1983, 473-496. C169, C173, C174, C178, C179 
[2] Barkley, D., Gomes, M. G. M. and Henderson, R. D., Three-dimensional instability in flow over a backward-facing step, J. Fluid Mech., 473, 2002, 167-190. http://dx.doi.org/10.1017/S002211200200232X C169

[3] Kaiktis, L., Karniadakis, G. E. and Orszag, S. A., Onset of three-dimensionality, equilibria, and early transition in flow over a backward-facing step, J. Fluid Mech., 231, 1991, 501-528. C169

[4] Kaiktis, L., Karniadakis, G. E. and Orszag, S. A., Unsteadiness and convective instabilities in two-dimensional flow over a backward-facing step, J. Fluid Mech., 321, 1996, 157-187. C169

[5] Sahin, M. and Owens, R. G., A numerical investigation of wall effects up to high blockage ratios on two-dimensional flow past a confined circular cylinder, Phys. Fluids, 16, 2004, 1305-1320. http://dx.doi.org/10.1063/1.1668285 C170, C178

[6] Sheard, G. S., Thompson, M. C. and Hourigan, K., From spheres to circular cylinders: classification of bluff ring transitions and structure of bluff ring wakes, J. Fluid Mech., 492, 2003, 147-180. http://dx.doi.org/10.1017/S0022112003005512 C172

[7] Thompson, M. C., Hourigan, K. and Sheridan, J., Three-dimensional instabilities in the wake of a circular cylinder, Exp. Therm. Fluid Sci., 12, 1996, 190-196.

http://dx.doi.org/10.1016/0894-1777(95)00098-4 C172

[8] Williams, P. T, and Baker, A. J., Numerical simulations of laminar flow over a 3D backward-facing step, Int. J. Numer. Methods Fluids, 24, 1997, 1159-1183.

http://www3.interscience.wiley.com/cgi-bin/abstract/61002259 C169, C173, C174, C178, C179 